\title{
Vector Space of Feynman Integrals and Multivariate Intersection Numbers
}

\author{
Hjalte Frellesvig, ${ }^{1,2 *}$ Federico Gasparotto, ${ }^{1,2, \dagger}$ Manoj K. Mandal, ${ }^{1,2, \ddagger}$ Pierpaolo Mastrolia®, ${ }^{1,2, \S}$ \\ Luca Mattiazzi, ${ }^{2,1, \uparrow}$ and Sebastian Mizera, ${ }^{3,4, *}$ \\ ${ }^{1}$ Dipartimento di Fisica e Astronomia, Università di Padova, Via Marzolo 8, 35131 Padova, Italy \\ ${ }^{2}$ INFN, Sezione di Padova, Via Marzolo 8, 35131 Padova, Italy \\ ${ }^{3}$ Perimeter Institute for Theoretical Physics, Waterloo, Ontario N2L 2Y5, Canada \\ ${ }^{4}$ Department of Physics and Astronomy, University of Waterloo, Waterloo, Ontario N2L 3G1, Canada
}

(Received 17 July 2019; published 12 November 2019)

\begin{abstract}
Feynman integrals obey linear relations governed by intersection numbers, which act as scalar products between vector spaces. We present a general algorithm for the construction of multivariate intersection numbers relevant to Feynman integrals, and show for the first time how they can be used to solve the problem of integral reduction to a basis of master integrals by projections, and to directly derive functional equations fulfilled by the latter. We apply it to the decomposition of a few Feynman integrals at one and two loops, as first steps toward potential applications to generic multiloop integrals. The proposed method can be more generally employed for the derivation of contiguity relations for special functions admitting multifold integral representations.
\end{abstract}

DOI: 10.1103/PhysRevLett.123.201602

Introduction.-Scattering amplitudes encode crucial information about collision phenomena in our Universe, from the smallest to the largest scales. Within the perturbative field-theoretical approach, the evaluation of multiloop Feynman integrals is mandatory for the determination of scattering amplitudes and related quantities. An exception is made for those cases where a limited number of kinematic invariants yields the use of direct integration techniques; the evaluation of multiloop Feynman integrals requires the exploitation of linear relations among integrals, in order to simplify the otherwise impossible calculations. Those relations can be used both for decomposing scattering amplitudes in terms of a basis of functions, referred to as master integrals (MIs), and for the evaluation of the latter. The standard procedure used to derive relations among Feynman integrals in dimensional regularization makes use of integration-by-parts identities (IBPs) [1], which are found by solving linear systems of equations [2] (see [3,4] and references therein for reviews). Algebraic manipulations in these cases are very demanding, and efficient algorithms for solving large-size systems of linear equations have been devised recently, by making use of finite field arithmetic and rational functions reconstruction [5-8].

In this Letter, we propose a novel, alternative approach for the direct decomposition of Feynman integrals. Our method

Published by the American Physical Society under the terms of the Creative Commons Attribution 4.0 International license. Further distribution of this work must maintain attribution to the author(s) and the published article's title, journal citation, and DOI. Funded by SCOAP ${ }^{3}$. is based on the identification of algebraic properties obeyed by Feynman integrals, not known until recently, and which we access by means of intersection theory.

In [9], it was shown that intersection numbers [10] of differential forms can be employed to define (what amounts to) a scalar product on a vector space of Feynman integrals in a given family. Using this approach, projecting any multiloop integral onto a basis of MIs is conceptually no different from decomposing a generic vector into a basis of a vector space. Within this new approach, relations among Feynman integrals can be derived avoiding the generation of intermediate, auxiliary expressions which are needed when applying Gauss' elimination, as in the standard IBPbased approaches. In the initial studies, $[9,11]$, this novel decomposition method was applied to the realm of special mathematical functions falling in the class of Lauricella $F_{D}$ functions, as well as to Feynman integrals on maximal cuts, i.e., with on-shell internal lines, mostly admitting a onefold integral representation. Those results concerned a partial construction of Feynman integral relations, mainly limited to the determination of the coefficients of the MIs with the same number of denominators as the decomposed integral, which was achieved by means of intersection numbers for univariate forms.

In this Letter, we make an important step further, and address the complete integral reduction, by determining all coefficients, including those associated to MIs corresponding to subgraphs. In the current Letter, we show its application to a few paradigmatic cases at one and two loops. Generic Feynman integrals admit multifold integral representations. Their complete decomposition requires the evaluation of intersection numbers for multivariate rational 
differential forms. Intersection numbers of multivariate forms have been previously studied in [12-20]. Recently, a new recursive algorithm was introduced in [21]. Here, we use its refined implementation and apply it to Feynman integrals, which provide a major step toward large-scale applicability of our strategy for the reduction to MIs. The results of this Letter show potential for further applications ranging from particle physics, through condensed matter and statistical mechanics, to gravitational-wave physics (while making new connections to mathematics).

Feynman integrals and differential forms.-Within the Baikov representation (BR), Feynman integrals can be cast in the form [22]

$$
I=\int_{\mathcal{C}} u(\mathbf{z}) \varphi(\mathbf{z})
$$

where the integration variables $\mathbf{z}=\left(z_{1}, \ldots, z_{n}\right)$ are the propagators of the diagrams, supplemented by a set of auxiliary propagators (related to the irreducible scalar products). In fact, the number $n$ of integration variables amounts to the number of scalar products formed by the external and the loop momenta. The function $u(\mathbf{z})$ is multivalued, and it is defined either as $u(\mathbf{z})=\mathcal{B}^{\gamma}(\mathbf{z})$ [23], or as $u(\mathbf{z})=\prod_{i} \mathcal{B}_{i}(\mathbf{z})^{\gamma_{i}}$ [24]. The factors $\mathcal{B}$ and $\mathcal{B}_{i}$ are graph (Baikov) polynomials; their exponents $(\gamma$ or $\gamma_{i} \notin \mathbb{Z}$ ) depend on the dimensional regulator $d$, and on the number of loops and external momenta of the corresponding diagram. The integration domain $\mathcal{C}$ is defined as such that, according to the case, either $\mathcal{B}$ or $\prod_{i} \mathcal{B}_{i}$ vanishes on its boundary $\partial \mathcal{C}$. In the integrand, $\varphi$ is a single-valued differential form and can generically be written as [25]

$$
\varphi(\mathbf{z})=\hat{\varphi}(\mathbf{z}) d^{n} \mathbf{z}, \quad \hat{\varphi}(\mathbf{z}) \equiv \frac{f(\mathbf{z})}{z_{1}^{a_{1}} \cdots z_{n}^{a_{n}}},
$$

with $d^{n} \mathbf{z} \equiv d z_{1} \wedge \ldots \wedge d z_{n}$, and where $a_{i} \in \mathbb{Z}$, and $f$ is a rational function of $\mathbf{z}$ with poles regulated by $u(\mathbf{z})$ [26]. Multiple-cut integrals [27,28], identified by the on-shell conditions $z_{i_{1}}=\ldots=z_{i_{k}}=0$, are also of the form (1), but their integrands depend on fewer integration variables (and their integration contour is modified); see [9,11].

Feynman integrals [and, more generally, hypergeometric integrals of the type in Eq. (1)], whose integrands differ by terms proportional to covariant derivatives, give the same result after integration. Employing Stokes' theorem, we find equivalence classes of $n$ forms, $\varphi \sim \varphi+\nabla_{\omega} \xi$, for any $(n-1)$-form $\xi$ and where $\nabla_{\omega} \equiv d+\omega \wedge$ is a covariant derivative with a one-form $\omega \equiv d \log u$, such that $\int_{\mathcal{C}} u \nabla_{\omega} \xi$ vanishes. The space of $n$-forms modulo the equivalence relation written above is a vector space known as twisted cohomology group $H_{\omega}^{n}$ [29]. We denote its elements by $\langle\varphi| \in H_{\omega}^{n}$. Within this framework, the Feynman integral $I$ from Eq. (1) can be interpreted as a pairing of $\langle\varphi|$ with the integration contour $\mid \mathcal{C}]$,

$$
I=\langle\varphi| \mathcal{C}]
$$

Consider a set of $\nu$ MIs, say $J_{i}$, defined as

$$
\left.J_{i}=\int_{\mathcal{C}} u(\mathbf{z}) e_{i}(\mathbf{z})=\left\langle e_{i}\right| \mathcal{C}\right], \quad i=1, \ldots, \nu,
$$

in terms of any independent set of differential forms $\left\langle e_{i}\right|$. Then, the decomposition of a generic integral $I$ in terms of the MIs $J_{i}$ can be interpreted as coming from the more fundamental decomposition of the differential form $\langle\varphi|$ in terms of the basis forms $\left\langle e_{i}\right|$, namely

$$
I=\sum_{i=1}^{\nu} c_{i} J_{i}, \quad \Leftrightarrow\langle\varphi|=\sum_{i=1}^{\nu} c_{i}\left\langle e_{i}\right|,
$$

with the coefficients determined by the master decomposition formula $[9,11]$

$$
c_{i}=\sum_{j=1}^{\nu}\left\langle\varphi \mid h_{j}\right\rangle\left(\mathbf{C}^{-1}\right)_{j i}, \quad \mathbf{C}_{i j}=\left\langle e_{i} \mid h_{j}\right\rangle,
$$

where $\left|h_{j}\right\rangle(j=1, \ldots, \nu)$ [42], span a dual (and auxiliary) vector space $\left(H_{\omega}^{n}\right)^{*}=H_{-\omega}^{n}$. The scalar product $\left\langle\varphi_{L} \mid \varphi_{R}\right\rangle$ between the two vector spaces is called intersection number of differential forms [10]. The characterization of the decomposition of Feynman integrals in terms of multivariate intersection numbers [12-21] is the main result of this Letter.

Using Eqs. (5) and (6), our algorithm for expressing any Feynman integral of the type of Eq. (1) as linear combinations of MIs proceeds along three steps as follows: 1. Determination of the number $\nu$ of MIs. 2. Choice of the bases of forms $\left\langle e_{i}\right|$ and $\left|h_{i}\right\rangle$. 3. Evaluation of the intersection numbers for multivariate forms, appearing in the entries of the $\mathbf{C}$ matrix, and in $\left\langle\varphi \mid h_{j}\right\rangle$.We finally remark that the coefficient $c_{i}$ in Eq. (6) is independent on the choice of the auxiliary basic forms $\left|h_{j}\right\rangle$ [43]. In the following, we choose $\hat{h}_{j}=\hat{e}_{j}$, namely $\left|h_{j}\right\rangle=\left|e_{j}\right\rangle$.

Number of master integrals.-Within the standard IBP decomposition, based on the so-called Laporta method, the number of MIs is determined at the end of the reduction procedure, by counting the irreducible integrals that are untouched by Gauss' elimination. The results of $[9,11,44-48]$ have been pointing to a geometrical characterization of the number $\nu$ of MIs, which, within our formalism, allow us to relate it to topological properties such as the dimension of the spaces $H_{ \pm \omega}^{n}$,

$$
\nu \equiv \operatorname{dim} H_{ \pm \omega}^{n}=(-1)^{n}\left[n+1-\chi\left(\mathcal{P}_{\omega}\right)\right],
$$

in terms of the Euler characteristic $\chi\left(\mathcal{P}_{\omega}\right)$ of the projective [49] variety $\mathcal{P}_{\omega}$, defined by $\mathcal{P}_{\omega}=\{$ set of poles of $\omega\}$, or 
equivalently by the submanifold where $u(z)$ vanishes or diverges (depending on signs of $\gamma_{i}$ ).

This connection yields the use of complex Morse (Picard-Lefschetz) theory to determine $\nu$ as the number of critical points of the function $\log u(\mathbf{z})$. Let us define

$$
\omega \equiv d \log u(\mathbf{z})=\sum_{i=1}^{n} \hat{\omega}_{i} d z_{i}
$$

then the number of critical points is given by the number of solutions of the system of equations

$$
\hat{\omega}_{i} \equiv \partial_{z_{i}} \log u(\mathbf{z})=0, \quad i=1, \ldots, n,
$$

with the short-hand notation $\partial_{z_{i}} \equiv \partial / \partial z_{i}$. Owing to the application of these novel mathematical concepts, the number $\nu$ can be accessed before the decomposition, as dictated by the geometrical properties of the graph (Baikov) polynomial associated to each Feynman diagram.

Multivariate intersection numbers.-A generic rational $n$ form can be decomposed into a combination of a one form and a $(n-1)$ form, as $\left\langle\varphi_{L}^{(\mathbf{n})}\right|=\sum_{i}\left\langle e_{i}^{(\mathbf{n}-\mathbf{1})}\right| \wedge\left\langle\varphi_{L, i}^{(n)}\right|$, $\left|\varphi_{R}^{(\mathbf{n})}\right\rangle=\sum_{j}\left|h_{j}^{(\mathbf{n}-\mathbf{1})}\right\rangle \wedge\left|\varphi_{R, j}^{(n)}\right\rangle$, where $i, j=1, \ldots, \nu_{\mathbf{n}-\mathbf{1}}$, with $\nu_{\mathbf{n}-\mathbf{1}}$ being the number of MIs in the inner space, spanned by the arbitrary bases $\left\langle e_{i}^{(\mathbf{n}-\mathbf{1})}|,| h_{j}^{(\mathbf{n}-\mathbf{1})}\right\rangle$. In the above expressions, $\left\langle\varphi_{L, i}^{(n)}\right|$ and $\left|\varphi_{R, j}^{(n)}\right\rangle$ are one forms (in the variable $z_{n}$ ), and they can be treated as coefficients of the basis expansion and hence obtained by projection. Then, $\mathbf{n}$-form intersection numbers can be evaluated recursively in terms of $(\mathbf{n}-\mathbf{1})$-form intersection numbers as [21]

${ }_{\mathbf{n}}\left\langle\varphi_{L}^{(\mathbf{n})} \mid \varphi_{R}^{(\mathbf{n})}\right\rangle=-\sum_{i=1}^{\nu_{\mathbf{n}-1}} \sum_{p \in \mathcal{P}_{n}} \operatorname{Res}_{z_{n}=p}\left({ }_{\mathbf{n}-\mathbf{1}}\left\langle\varphi_{L}^{(\mathbf{n})} \mid h_{i}^{(\mathbf{n}-\mathbf{1})}\right\rangle \psi_{i}^{(n)}\right)$,

where the functions $\psi_{i}^{(n)}$ are the solutions of the system of differential equations

$$
\partial_{z_{n}} \psi_{i}^{(n)}-\sum_{j=1}^{\nu_{\mathrm{n}-1}} \hat{\boldsymbol{\Omega}}_{i j}^{(n)} \psi_{j}^{(n)}=\hat{\varphi}_{R, i}^{(n)} ;
$$

the elements of the $\hat{\boldsymbol{\Omega}}^{(n)}$ matrix are given by

$\hat{\boldsymbol{\Omega}}_{i j}^{(n)}=-\sum_{k=1}^{\nu_{\mathbf{n}-1}}\left(\mathbf{C}_{(\mathbf{n}-\mathbf{1})}^{-1}\right)_{i k \mathbf{n}-\mathbf{1}}\left\langle e_{k}^{(\mathbf{n}-\mathbf{1})} \mid\left(\partial_{z_{n}}-\hat{\omega}_{n}\right) h_{j}^{(\mathbf{n}-\mathbf{1})}\right\rangle$,

and $\mathcal{P}_{n}$ is the set of poles of $\hat{\boldsymbol{\Omega}}^{(n)}$ (including possible poles at infinity). Additional mathematical details are provided in the Supplemental Material [50] for the interested readers.

Integral decomposition: bottom-up approach.-For a given integral, any set of denominators identifies a sector.
Therefore, any cut (a set of vanishing denominators) corresponds to a sector. The number of MIs can be determined by counting the number of critical points, according to Eq. (9): we observed that using $u(\mathbf{z})$ on the cut, it provides the number of MIs in the corresponding sector, whereas using $u(\mathbf{z})$ on the cut with regulating factors $z_{i}^{\rho_{i}}$ for any uncut denominator, it yields the number of MIs surviving on that cut.

After determining the number of MIs, the decomposition of Feynman integrals can be obtained with Eq. (6). The determination of coefficients can be performed on unitarity cuts, where the integrands are simpler, and the evaluation of the multivariate intersections requires fewer iterations. A minimal set of spanning cuts is sufficient to retrieve the information of the complete decomposition [55], and then, using the regulated $u(\mathbf{z})$, the master decomposition formula (6) yields the coefficients of those MIs that survive on the cut (after taking $\rho_{i} \rightarrow 0$ limit). As in the case of IBPbased approaches, additional relations may be obtained from the symmetries of the diagrams, in order to minimize the number of independent integrals.

As discussed in Refs. $[9,11]$ also, differential equations in kinematic variables, e.g., $\partial_{s} J_{i}=\sum_{j} a_{i j} J_{j}$, and dimensional recurrence relations for MIs can be obtained with the above techniques.

Massless box diagram.-Let us consider the massless box diagram at one loop, Fig. 1. Within the BR,

$$
\begin{aligned}
u(\mathbf{z})= & \left\{\left(s t-s z_{4}-t z_{3}\right)^{2}-2 t z_{1}\left[s\left(t+2 z_{3}-z_{2}-z_{4}\right)+t z_{3}\right]\right. \\
& \left.+s^{2} z_{2}^{2}+t^{2} z_{1}^{2}-2 s z_{2}\left[t\left(s-z_{3}\right)+z_{4}(s+2 t)\right]\right\}^{(d-5) / 2} .
\end{aligned}
$$

For each of the $15\left(=2^{4}-1\right)$ sectors, we use Eq. (9) on the corresponding cut to determine the number $N_{\text {sector }}$ of MIs. The nonzero cases are [56] $N_{\{1,2,3,4\}}=1, N_{\{1,3\}}=1$, $N_{\{2,4\}}=1$, amounting to three MIs. We choose them to be

$$
J_{1}=\square, \quad J_{2}=\chi \chi, \quad J_{3}=\bigvee,
$$

so that any integral $I$ of the form of Eq. (1), with $u$ given in Eq. (13), and $\varphi$ defined in Eq. (2) (with $n=4$ ), can be decomposed as

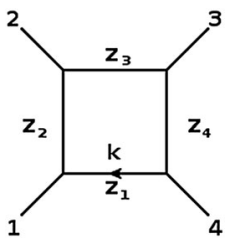

FIG. 1. Massless box with massless external legs: $p_{i}^{2}=0$, for $i=1,2,3,4$, with $s=\left(p_{1}+p_{2}\right)^{2}$ and $t=\left(p_{2}+p_{3}\right)^{2}$. 


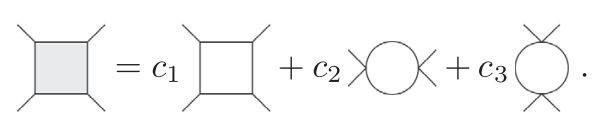

We determine the set of spanning cuts as $\left(\mathrm{Cut}_{\{1,3\}}, \mathrm{Cut}_{\{2,4\}}\right)$ to obtain the full decomposition. (i) On the $\mathrm{Cut}_{\{1,3\}}$, we use the regularized $u_{1,3}=z_{2}^{\rho_{2}} z_{4}^{\rho_{4}} u\left(0, z_{2}, 0, z_{4}\right)$ to obtain the corresponding $\hat{\omega}_{2}$ and $\hat{\omega}_{4}$. After choosing the $z_{4}$ coordinate as the inner space, using Eq. (9), we get $\nu_{(42)}=2$, and $\nu_{(4)}=2$. Accordingly, we choose the basis forms

$\hat{e}_{1}^{(42)}=\frac{1}{z_{2} z_{4}}, \quad \hat{e}_{2}^{(42)}=1, \quad \hat{e}_{1}^{(4)}=\frac{1}{z_{4}}, \quad \hat{e}_{2}^{(4)}=1$.

(ii) $\mathrm{Cut}_{\{2,4\}}$ can be obtained from $\mathrm{Cut}_{\{1,3\}}$, because of the symmetry of the graph under the exchange of $s$ and $t$, with the substitution of $\left(z_{4}, z_{2}\right) \rightarrow\left(z_{3}, z_{1}\right)$ and the space labels $(42) \rightarrow(31)$ and $(4) \rightarrow(3)$.

With the help of Eq. (6) and using the multivariate intersection numbers for two forms, we determine the coefficients $c_{i}$ in Eq. (15).

Example.-Let us illustrate the decomposition of

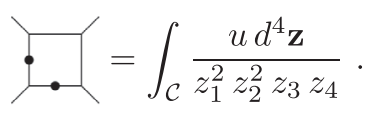

On the $\mathrm{Cut}_{\{1,3\}}$, we obtain

$$
\stackrel{-}{=} \int_{\mathcal{C}} u_{1,3} \varphi_{1,3}, \quad \varphi_{1,3}=\hat{\varphi}_{1,3} d z_{4} \wedge d z_{2},
$$

where $\hat{\varphi}_{1,3}=\left[\hat{\omega}_{1} /\left(z_{2}^{2} z_{4}\right)\right]$. On this specific cut, we have

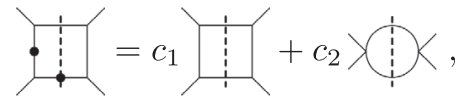

with

$$
\begin{aligned}
c_{1} & =\sum_{j=1}^{2}\left\langle\varphi_{1,3} \mid e_{j}^{(42)}\right\rangle\left(\mathbf{C}_{(42)}^{-1}\right)_{j 1}=\frac{(d-6)(d-5)}{s t}, \\
c_{2}=\sum_{j=1}^{2}\left\langle\varphi_{1,3} \mid e_{j}^{(42)}\right\rangle\left(\mathbf{C}_{(42)}^{-1}\right)_{j 2} & =-\frac{4(d-5)(d-3)}{s^{3} t} .
\end{aligned}
$$

On the $\mathrm{Cut}_{\{2,4\}}$, we have

$$
\left.\stackrel{\longrightarrow}{\cdots}=c_{1}\right)
$$

where we find $c_{1}$ in agreement with Eq. (20) and $c_{3}=\left.c_{2}\right|_{s \leftrightarrow t}$. Finally, the integral of Eq. (17) is decomposed in terms

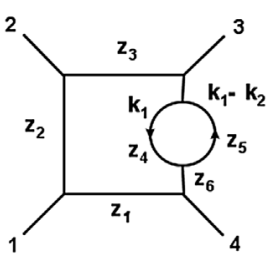

FIG. 2. Massless box with a self-energy insertion diagram: $p_{i}^{2}=0$, for $i=1,2,3,4$, with $s=\left(p_{1}+p_{2}\right)^{2}$ and $t=$ $\left(p_{2}+p_{3}\right)^{2}$.

of MIs, as in Eq. (15), in agreement with the IBP decomposition.

A two-loop diagram.-Let us consider the massless box with a self-energy insertion diagram in Fig. 2.

Within the loop-by-loop BR [24],

$$
u(\mathbf{z})=\mathcal{B}_{1}^{\frac{2-d}{2}} \mathcal{B}_{2}^{\frac{d-3}{2}} \mathcal{B}_{3}^{\frac{d-5}{2}},
$$

where

$$
\begin{aligned}
\mathcal{B}_{1}= & z_{6}, \quad \mathcal{B}_{2}=2\left(z_{5}+z_{6}\right) z_{4}-z_{4}^{2}-\left(z_{5}-z_{6}\right)^{2}, \\
\mathcal{B}_{3}= & t^{2} z_{1}^{2}+s^{2} z_{2}^{2}-2 t z_{1}\left[(2 s+t) z_{3}+s\left(t-z_{2}-z_{6}\right)\right] \\
& -2 s z_{2}\left[s t-t z_{3}+(s+2 t) z_{6}\right]+\left[t z_{3}+s\left(z_{6}-t\right)\right]^{2} .
\end{aligned}
$$

For all the possible $63\left(=2^{6}-1\right)$ sectors, using Eq. (9) on the corresponding cut, we determine the number $N_{\text {sector }}$ of MIs. The nonzero cases are $N_{1,2,3,4,5}=1, N_{1,3,4,5}=1$, and $N_{2,4,5}=1$, giving three MIs. We choose them as

$$
J_{1}={ }_{Q}, J_{2}=\chi, \quad J_{3}=\chi .
$$

Any integral $I$ of the form (1), with $u$ given in (22), and $\varphi$ defined in Eq. (2) (with $n=6$ ), can be decomposed as

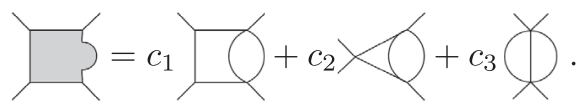

We use the set of spanning cuts $\left(\mathrm{Cut}_{\{1,3,4,5\}}, \mathrm{Cut}_{\{2,4,5\}}\right)$ to obtain the full decomposition. (i) On the $\mathrm{Cut}_{\{1,3,4,5\}}$, we use the regularized $u_{1,3,4,5}=z_{2}^{\rho_{2}} u\left(0, z_{2}, 0,0,0, z_{6}\right)$ to obtain the corresponding $\hat{\omega}_{2}$ and $\hat{\omega}_{6}$. After choosing the $z_{2}$ coordinate for the inner space, using Eq. (9), we get $\nu_{(26)}=2$ (amounting to the number of solutions of $\hat{\omega}_{2}=0$ and $\hat{\omega}_{6}=0$ ) and $\nu_{(2)}=2$ (amounting to the number of solutions of $\hat{\omega}_{2}=0$ ). We choose the basis forms as

$$
\hat{e}_{1}^{(26)}=\hat{e}_{1}^{(2)}=\frac{1}{z_{2}}, \quad \hat{e}_{2}^{(26)}=\hat{e}_{2}^{(2)}=1 .
$$

(ii) On the cut $\operatorname{Cut}_{\{2,4,5\}}$, we use the regularized $u_{2,4,5}=$ $z_{1}^{\rho_{1}} z_{3}^{\rho_{3}} u\left(z_{1}, 0, z_{3}, 0,0, z_{6}\right)$ to obtain the corresponding $\hat{\omega}_{1}$, 
$\hat{\omega}_{3}$, and $\hat{\omega}_{6}$. Using Eq. (9), we get $\nu_{(136)}=2$ (amounting to the number of solutions of $\hat{\omega}_{1}=0, \hat{\omega}_{3}=0$, and $\hat{\omega}_{6}=0$ ), $\nu_{(13)}=2$ (amounting to the number of solutions of $\hat{\omega}_{1}=0$ and $\hat{\omega}_{3}=0$ ), and $\nu_{(1)}=2$ (amounting to the number of solutions of $\hat{\omega}_{1}=0$ ). The basis forms are chosen as

$$
\begin{aligned}
\hat{e}_{1}^{(136)} & =\frac{1}{z_{1} z_{3}}, \quad \hat{e}_{2}^{(136)}=1, \quad \hat{e}_{1}^{(13)}=\hat{e}_{1}^{(1)}=z_{1}, \\
\hat{e}_{2}^{(13)} & =\hat{e}_{2}^{(1)}=1 .
\end{aligned}
$$

The basic forms defined above can be used for the evaluation of the multivariate intersection numbers, required to determine the coefficients of the complete integral decomposition.

Example.-Let us consider the decomposition of

$$
\zeta=\int_{\mathcal{C}} \frac{u d^{6} \mathbf{z}}{z_{1} z_{2}^{2} z_{3} z_{4} z_{5} z_{6}^{2}}
$$

On the $\mathrm{Cut}_{\{1,3,4,5\}}$, we obtain

$$
:=\int_{\mathcal{C}} u_{1,3,4,5} \hat{\varphi}_{1,3,4,5} d z_{2} \wedge d z_{6}
$$

where $\hat{\varphi}_{1,3,4,5}=\left[\hat{\omega}_{2} /\left(z_{2} z_{6}^{2}\right)\right]$. On this specific cut, we have

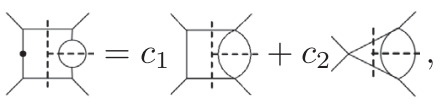

where the coefficients can be obtained from two-form intersections as

$$
c_{i}=\sum_{j=1}^{2}\left\langle\varphi_{1,3,4,5} \mid e_{j}^{(26)}\right\rangle\left(\mathbf{C}_{(26)}^{-1}\right)_{j i} .
$$

On the $\mathrm{Cut}_{\{2,4,5\}}$, we obtain

$$
\underset{\mathcal{C}}{\longrightarrow} \int_{\mathcal{C}} u_{2,4,5} \hat{\varphi}_{2,4,5} d z_{1} \wedge d z_{3} \wedge d z_{6}
$$

where $\hat{\varphi}_{2,4,5}=\left[\hat{\omega}_{2} /\left(z_{1} z_{3} z_{6}^{2}\right)\right]$.

On this specific cut, we have

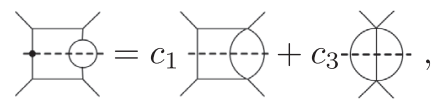

where the coefficients can be obtained from three-form intersections as

$$
\begin{aligned}
& c_{1}=\sum_{j=1}^{2}\left\langle\varphi_{2,4,5} \mid e_{j}^{(136)}\right\rangle\left(\mathbf{C}_{(136)}^{-1}\right)_{j 1}, \\
& c_{3}=\sum_{j=1}^{2}\left\langle\varphi_{2,4,5} \mid e_{j}^{(136)}\right\rangle\left(\mathbf{C}_{(136)}^{-1}\right)_{j 2},
\end{aligned}
$$

yielding $c_{1}$ in agreement with the value found on the $\mathrm{Cut}_{\{1,3,4,5\}}$. Finally, we obtain a relation of the type in Eq. (25), in agreement with the IBP decomposition.

Beside the shown examples, we have successfully applied the aforementioned algorithm to the complete decomposition of a few one- and two-loop integrals involving the evaluation of up to six-variable intersection numbers. The resulting expressions are in agreement with the IBP relations [57-60].

Conclusions. - In this Letter, we exposed a novel vector space structure for Feynman integrals. Elaborating on the original proposal of [9] and on the wider studies [11,21], we have shown that Feynman integrals can be expressed in terms of a complete basis of integrals, by making use of intersection numbers, which act as scalar products for the vector space of integrals, through the pairing of differential forms appearing in their integrands. Let us notice that the result of the multivariate intersection numbers should not depend on the ordering of the integration variables, chosen in the recursive formula. Nevertheless, we observed that suitably chosen variable orderings may simplify and fasten the recursive procedure. This is a feature of the proposed algorithm that requires a dedicated study, which goes beyond the goal of the present work. Within Baikov representation, one-loop and multiloop integrands have a similar structure, and therefore we expect that our decomposition algorithm can be applied to integrals associated with more complex diagrams than the ones considered here, which we plan to investigate in the near future. Additional interesting applications may involve the evaluation of master integrals by differential equations, using intersection theory.

Scattering amplitudes are analytic functions, determined by their singularities. Intersection numbers, and their relation to Stokes' and Cauchy's residue theorems, embed what we believe is a clean role of analyticity in the amplitudes decomposition. We investigated the geometric origin of master integrals and their relations to the number of critical points and Euler characteristics. Applications to Feynman integrals in representations other than Baikov will also constitute topics of future works. The present study can be broadly applied in the context of theoretical particle physics, condensed matter and statistical mechanics, gravitational-wave physics, as well as mathematics.

We would like to thank Janko Boehm, Thibault Damour, Maxim Kontsevich, Stefano Laporta, and Giovanni Ossola for useful discussions and comments. H. F., F. G., M. K. M., and P.M. wish to acknowledge the organizers and the participants of The Mathematics of Linear Relations between Feynman Integrals, Mainz Institute for Theoretical Physics, 
Johannes Gutenberg University. CloudVeneto is acknowledged for the use of computing and storage facilities. The work of F. G., M. K. M., and P. M. is supported by the Supporting TAlent in ReSearch at Padova University (UniPD STARS Grant 2017 "Diagrammalgebra"). The work of H. F. is part of the HiProLoop project funded by the European Union's Horizon 2020 research and innovation programme under the Marie Skłodowska-Curie Grant No. 747178. Research at Perimeter Institute is supported in part by the Government of Canada through the Department of Innovation, Science and Economic Development Canada and by the Province of Ontario through the Ministry of Economic Development, Job Creation and Trade. The figures were drawn with Jaxodraw [61] based on Axodraw [62].

*hjalte.frellesvig@pd.infn.it

†ederico.gasparotto@pd.infn.it

*manojkumar.mandal@pd.infn.it

\$pierpaolo.mastrolia@pd.infn.it

luca.mattiazzi@pd.infn.it

smizera@pitp.ca

[1] K. G. Chetyrkin and F. V. Tkachov, Integration by parts: The algorithm to calculate beta functions in 4 loops, Nucl. Phys. B192, 159 (1981).

[2] S. Laporta, High precision calculation of multiloop Feynman integrals by difference equations, Int. J. Mod. Phys. A 15, 5087 (2000).

[3] A. G. Grozin, Integration by parts: An introduction, Int. J. Mod. Phys. A 26, 2807 (2011).

[4] Y. Zhang, Lecture notes on multi-loop integral reduction and applied algebraic geometry, arXiv:1612.02249.

[5] A. von Manteuffel and R. M. Schabinger, A novel approach to integration by parts reduction, Phys. Lett. B 744, 101 (2015).

[6] T. Peraro, Scattering amplitudes over finite fields and multivariate functional reconstruction, J. High Energy Phys. 12 (2016) 030.

[7] J. Klappert and F. Lange, Reconstructing rational functions with FireFly, arXiv:1904.00009.

[8] T. Peraro, FiniteFlow: Multivariate functional reconstruction using finite fields and dataflow graphs, J. High Energy Phys. 07 (2019) 031.

[9] P. Mastrolia and S. Mizera, Feynman integrals and intersection theory, J. High Energy Phys. 02 (2019) 139.

[10] K. Cho and K. Matsumoto, Intersection theory for twisted cohomologies and twisted Riemann's period relations I, Nagoya mathematical Journal 139, 67 (1995).

[11] H. Frellesvig, F. Gasparotto, S. Laporta, M. K. Mandal, P. Mastrolia, L. Mattiazzi, and S. Mizera, Decomposition of Feynman integrals on the maximal cut by intersection numbers, J. High Energy Phys. 05 (2019) 153.

[12] K. Matsumoto, Quadratic identities for hypergeometric series of type $(k, l)$, Kyushu J. Math. 48, 335 (1994).

[13] K. Matsumoto, Intersection numbers for logarithmic $k$-forms, Osaka Journal of mathematics 35, 873 (1998).
[14] K. Ohara, Y. Sugiki, and N. Takayama, Quadratic relations for generalized hypergeometric functions ${ }_{p} F_{p-1}$, Funkcialaj ekvacioj Serio internacia 46, 213 (2003).

[15] Y. Goto, Twisted cycles and twisted period relations for Lauricella's hypergeometric function $F_{C}$, Int. J. Math. 24, 1350094 (2013).

[16] Y. Goto and K. Matsumoto, The monodromy representation and twisted period relations for Appell's hypergeometric function $F_{4}$, Nagoya mathematical Journal 217, 61 (2015)

[17] Y. Goto, Twisted period relations for Lauricella's hypergeometric functions $F_{A}$, Osaka Journal of mathematics 52, 861 (2015).

[18] Y. Goto, Intersection numbers and twisted period relations for the generalized hypergeometric function ${ }_{m+1} F_{m}$, Kyushu J. Math. 69, 203 (2015).

[19] S. Mizera, Scattering Amplitudes from Intersection Theory, Phys. Rev. Lett. 120, 141602 (2018).

[20] S.-J. Matsubara-Heo and N. Takayama, An algorithm of computing cohomology intersection number of hypergeometric integrals, arXiv:1904.01253.

[21] S. Mizera, Aspects of scattering amplitudes and moduli space localization, arXiv:1906.02099.

[22] A prefactor $K$, depending on $d$ and on external kinematic invariants, is dropped while deriving integral relations. However, it is considered for the construction of differential equations and dimensional recurrence relations as discussed in Ref. [11].

[23] P. A. Baikov, Explicit solutions of the multiloop integral recurrence relations and its application, Nucl. Instrum. Methods Phys. Res., Sect. A 389, 347 (1997).

[24] H. Frellesvig and C. G. Papadopoulos, Cuts of Feynman integrals in Baikov representation, J. High Energy Phys. 04 (2017) 083.

[25] Here and in the following, $\hat{\varphi}$ denotes the differentialstripped version of the form $\varphi$, that is $\varphi=\hat{\varphi} d^{n} \mathbf{z}$.

[26] Within our formalism, one needs to include regulating factors for all singularities in order remove surface terms in two cases: either $z_{i}^{\rho_{i}}$ for each pole of $\varphi(\mathbf{z})$ not present in $u(\mathbf{z})$, or $\mathcal{B}_{i}(\mathbf{z})^{\rho_{i}}$ whenever $\gamma_{i} \in \mathbb{Z}$. In both cases, $\rho_{i} \notin \mathbb{Z}$ are sent to zero at the end of the computation.

[27] Z. Bern, L. J. Dixon, D. C. Dunbar, and D. A. Kosower, One loop $\mathrm{n}$ point gauge theory amplitudes, unitarity and collinear limits, Nucl. Phys. B425, 217 (1994).

[28] Z. Bern, L. J. Dixon, D. C. Dunbar, and D. A. Kosower, Fusing gauge theory tree amplitudes into loop amplitudes, Nucl. Phys. B435, 59 (1995).

[29] We refer the interested reader to [21,30,31] for reviews of twisted (co)homologies and their intersection theory, as well as $[9,11,19,21,32-36]$ and [38-41] for some recent applications of these ideas to physics.

[30] K. Aomoto and M. Kita, Theory of Hypergeometric Functions, Springer Monographs in Mathematics (Springer, Japan, 2011).

[31] M. Yoshida, Hypergeometric Functions, My Love: Modular Interpretations of Configuration Spaces, Aspects of Mathematics (Vieweg+Teubner Verlag, Wiesbaden, 2013).

[32] S. Mizera, Combinatorics and topology of Kawai-LewellenTye relations, J. High Energy Phys. 08 (2017) 097. 
[33] K. Mimachi and M. Yoshida, Intersection numbers of twisted cycles and the correlation functions of the conformal field theory, Commun. Math. Phys. 234, 339 (2003).

[34] K. Mimachi and M. Yoshida, Intersection numbers of twisted cycles associated with the Selberg integral and an application to the conformal field theory, Commun. Math. Phys. 250, 23 (2004).

[35] H. Frost, Biadjoint scalar tree amplitudes and intersecting dual associahedra, J. High Energy Phys. 06 (2018) 153.

[36] Z. Li and C. Zhang, Moduli space of paired punctures, cyclohedra and particle pairs on a circle, J. High Energy Phys. 05 (2019) 029.

[37] F. Brown and C. Dupont, Single-valued integration and superstring amplitudes in genus zero, arXiv:1810.07682.

[38] L. de la Cruz, A. Kniss, and S. Weinzierl, Properties of scattering forms and their relation to associahedra, J. High Energy Phys. 03 (2018) 064.

[39] S. Abreu, R. Britto, C. Duhr, and E. Gardi, Algebraic Structure of Cut Feynman Integrals and the Diagrammatic Coaction, Phys. Rev. Lett. 119, 051601 (2017).

[40] S. Abreu, R. Britto, C. Duhr, E. Gardi, and J. Matthew, Coaction for Feynman integrals and diagrams, Proc. Sci., LL2018 (2018) 047 [arXiv:1808.00069].

[41] L. de la Cruz, Feynman integrals as A-hypergeometric functions, arXiv:1907.00507.

[42] Suitable choices of orthonormal basis forms can be made $[9,11]$, such that metric matrix becomes the identity matrix, $\mathbf{C}_{i j}=\delta_{i j}$, yielding a simplified master decomposition formula Eq. (6).

[43] The invariance of $c_{i}$ for different choices of dual basic forms $h_{j}$ constitutes a sanity check of the result.

[44] A. V. Smirnov and A. V. Petukhov, The number of master integrals is finite, Lett. Math. Phys. 97, 37 (2011).

[45] R. N. Lee and A. A. Pomeransky, Critical points and number of master integrals, J. High Energy Phys. 11 (2013) 165.

[46] P. Aluffi and M. Marcolli, Feynman motives of banana graphs, Commun. Theor. Phys. 3, 1 (2009).

[47] M. Marcolli, Motivic renormalization and singularities, Clay Math. Proc. 11, 409 (2010).

[48] T. Bitoun, C. Bogner, R. P. Klausen, and E. Panzer, Feynman integral relations from parametric annihilators, Lett. Math. Phys. 109, 497 (2019).
[49] Defining $\mathcal{P}_{\omega}$ projectively ensures that all points at infinity are taken into account.

[50] See Supplemental Material at http://link.aps.org/ supplemental/10.1103/PhysRevLett.123.201602 for an extended discussion on counting the number of MIs, computing multivariate intersection numbers, and additional examples, which includes Refs. [9-11,19,21,24,33-36,47, 51-54].

[51] K. Aomoto, On vanishing of cohomology attached to certain many valued meromorphic functions, J. Math. Soc. Jpn. 27, 248 (1975).

[52] J. Milnor, Morse Theory (AM-51), in Annals of Mathematics Studies No. v. 51 (Princeton University Press, Princeton, 2016).

[53] E. Witten, Analytic continuation of Chern-Simons theory, AMS/IP Stud. Adv. Math. 50, 347 (2011).

[54] R. Silvotti, On a conjecture of Varchenko, Inventiones Mathematicae 126, 235 (1996).

[55] K. J. Larsen and Y. Zhang, Integration-by-parts reductions from unitarity cuts and algebraic geometry, Phys. Rev. D 93, 041701(R) (2016).

[56] If the Baikov polynomial $\mathcal{B}$ is a nonzero constant on the maximal cut, the integral is fully localized by the cut conditions. In this case, the condition $\omega=0$ is always satisfied, and there is $\nu=1$ master integral.

[57] A. V. Smirnov, FIRE5: A C++ implementation of Feynman Integral REduction, Comput. Phys. Commun. 189, 182 (2015).

[58] R. N. Lee, Presenting LiteRed: A tool for the Loop InTEgrals REDuction, arXiv:1212.2685.

[59] A. von Manteuffel and C. Studerus, Reduze 2-distributed Feynman integral reduction, arXiv:1201.4330.

[60] P. Maierhöfer, J. Usovitsch, and P. Uwer, Kira-A Feynman integral reduction program, Comput. Phys. Commun. 230, 99 (2018).

[61] D. Binosi, J. Collins, C. Kaufhold, and L. Theussl, JaxoDraw: A graphical user interface for drawing Feynman diagrams. Version 2.0 release notes, Comput. Phys. Commun. 180, 1709 (2009).

[62] J. A. M. Vermaseren, Axodraw, Comput. Phys. Commun. 83, 45 (1994). 